\title{
Point-Based Elastic Registration of Medical Image Data Using Approximating Thin-Plate Splines
}

\author{
K. Rohr ${ }^{1}$, H.S. Stiehl ${ }^{1}$, R. Sprengel ${ }^{1}$, W. Beil ${ }^{1}$, \\ T.M. Buzug ${ }^{2}$, J. Weese ${ }^{2}$, and M.H. Kuhn ${ }^{2}$ \\ 1 Universität Hamburg, Fachbereich Informatik, Arbeitsbereich Kognitive Systeme, \\ Vogt-Kölln-Str. 30, D-22527 Hamburg, Germany \\ 2 Philips Research, Technical Systems Hamburg, Röntgenstr. 24-26, \\ D-22335 Hamburg, Germany
}

\begin{abstract}
We consider elastic registration of medical image data based on thin-plate splines using a set of corresponding anatomical point landmarks. Previous work on this topic has concentrated on using interpolation schemes. Such schemes force the corresponding landmarks to exactly match each other and assume that the landmark positions are known exactly. However, in real applications the localization of landmarks is always prone to some error. Therefore, to take into account these localization errors, we have investigated the application of an approximation scheme which is based on regularization theory. This approach generally leads to a more accurate and robust registration result. In particular, outliers do not disturb the registration result as much as is the case with an interpolation scheme. Also, it is possible to individually weight the landmarks according to their localization uncertainty. In addition to this study, we report on investigations into semi-automatic extraction of anatomical point landmarks.
\end{abstract}

\section{Introduction}

In neurosurgery and radiotherapy it is important to either register images from different modalities, e.g. CT (X-ray Computed Tomography) and MR (Magnetic Resonance) images, or to match images to atlas representations. If only rigid transformations were applied, then the accuracy of the resulting match often is not satisfactory w.r.t. clinical requirements. In general, nonrigid transformations are required to cope with the variations between the data sets. A special class of general nonrigid transformations are elastic transformations which allow for local adaptivity and are constrained to some kind of continuity or smoothness.

This contribution is concerned with elastic registration of medical image data based on a set of corresponding anatomical landmarks. Such a feature-based approach comprises three steps: (1) Extraction of landmarks in the different data sets, (2) Establishing the correspondence between the landmarks, and (3) Computing the transformation between the data sets using the information from (1) and (2). Among the different types of landmarks (points, lines, surfaces, and volumes) we here consider point landmarks. 
Previous work on point-based elastic registration has concentrated on i) selecting the corresponding landmarks manually and on ii) using an interpolating transformation model (Bookstein [2], Evans et al. [6], and Mardia and Little [10]). The basic approach draws upon thin-plate splines and is computationally efficient, robust, and general w.r.t. different types of images and atlases. Also, the approach is well-suited for user-interaction which is important in clinical scenaria. However, an interpolation scheme forces the corresponding landmarks to exactly match each other. The underlying assumption is that the landmark positions are known exactly. In real applications, however, the localization of landmarks is always prone to some error. This is true for interactive as well as for automatic landmark localization. Therefore, to take into account these localization errors, we have investigated the application of an approximation scheme where the corresponding thin-plate splines result from regularization theory. Generally, such an approach yields a more accurate and robust registration result. In particular, outliers do not disturb the registration result as much as is the case with an interpolation scheme. Also, it is possible to individually weight the landmarks according to their localization uncertainty. We have applied this approach to elastic registration of 2D tomographic images of the human brain. Investigations for 3D images are under way. Additionally, we report on investigations toward the automatic extraction of anatomical point landmarks using differential operators. We will present first experimental results on 2D and 3D tomographic images. Algorithms for this task are important since interactive selection of landmarks is time-consuming and often lacks accuracy.

In the following, we first discuss clinical applications of point-based elastic registration. Then, we briefly review the original thin-plate interpolation scheme and extend this approach to an approximation scheme. Finally, we describe investigations into semi-automatic landmark localization.

\section{Clinical Applications for Elastic Registration}

Although elastic matching is not routinely used in clinical practice yet, there are several application scenaria in which elastic matching is believed to improve current therapeutical procedures.

\subsection{Image-atlas matching}

One possible application is trajectory planning for neurosurgical intervention. Pain treatment as well as epilepsy treatment sometimes require to localize a functionally important region not visible in the available image data. There are instructions available in the literature how to construct the position of such a region given landmarks which can be identified in CT or MR images. Hence, it is useful to superimpose an atlas with a medical image as already proposed by Talairach. Due to the individual variability of anatomical structures, rigid registration is generally not sufficient and elastic matching should be applied. 


\subsection{CT-MR matching}

Another application is the registration of CT and MR images for the purpose of radiotherapy planning. Additionally, a template atlas can be superimposed on the MR image to indicate, for example, organs at risk. This superposition result is then overlayed on the CT image prior to dose calculation and isodose visualization on the MR image. It is questionable whether rigid registration is suitable for this purpose since MR images are geometrically distorted. On the one hand, scanner-induced distortions have to be coped with which are caused by, e.g., inhomogeneities of the main magnetic field, imperfect slice or volume selection pulses, nonlinearities of the magnetic field gradients, and eddy currents [11]. These distortions can be reduced by suitable calibration steps: The inhomogeneities of the main magnetic field are minimized by passive and active shimming whereas, e.g., the gradient nonlinearities cannot be completely shimmed. Thus, depending on the scanner protocol, the sum of all remaining distortions leads to a residual error of a few millimeters (for a spherical field of view of $25 \mathrm{~cm}$ ). On the other hand, there are geometrical distortions in MR images that are induced by the patient and cannot be removed by calibration. Parameters such as susceptibility variations, chemical shift for non-water protons and flow-induced distortions for vessels are very important. While the susceptibility difference of tissue and bone is negligible, the susceptibility difference between tissue and air is approximately $10^{-5}$. This can result in a field variation of up to $10 \mathrm{ppm}$ and geometrical distortions of more than $5 \mathrm{~mm}$ [9],[4] which is most important for the nasal and aural regions. Consequently, due to the scanner- as well as the patient-induced distortions of the MR image CT and MR images cannot be satisfactorily registered using a rigid transformation.

\section{Thin-Plate Spline Interpolation}

The use of thin-plate spline interpolation for point-based elastic registration of medical images was first proposed by Bookstein [2]. Here, we briefly describe this method in the general context of $d$-dimensional images (see also [15]): Given two landmark sets each consisting of $n$ landmarks $\mathbf{p}_{i}$ and $\mathbf{q}_{i}, i=1, \ldots, n$ in two images of dimension $d$, find the transformation $\mathbf{u}$ within a suitable Hilbert-space $H^{d}$ of admissible functions, which i) minimizes a given functional $J: H^{d} \rightarrow \mathbb{R}$ and ii) fulfills the interpolation conditions

$$
\mathbf{u}\left(\mathbf{p}_{i}\right)=\mathbf{q}_{i}, \quad i=1, \ldots, n .
$$

We only consider such functionals $J(\mathbf{u})$ which can be separated into a sum of similar functionals that only depend on one component of the transformation $\mathbf{u}$. Thus, the problem of finding $\mathbf{u}$ can be subdivided into $d$ problems for each component $z$ of $\mathbf{u}$. In the case of interpolation the functional is fully described through the dimension $d$ of the domain and the order $m$ of derivatives used [5] and can be written as

$$
J_{m}^{d}(z)=\sum_{\alpha_{1}+\ldots+\alpha_{d}=m} \frac{m !}{\alpha_{1} ! \cdots \alpha_{d} !} \int_{\mathbb{R}^{d}}\left(\frac{\partial^{m} z}{\partial x_{1}^{\alpha_{1}} \cdots \partial x_{d}^{\alpha_{d}}}\right)^{2} d \mathbf{x} .
$$


The functional is invariant w.r.t. translations and rotations.

Let a set of functions $\phi_{i}$ span the space $\Pi^{m-1}\left(\mathbb{R}^{d}\right)$ of all polynomials on $\mathbb{R}^{d}$ up to order $m-1$, which is the nullspace of $J_{m}^{d}$. The dimension of this space is $M=(d+m-1) ! /(d !(m-1) !)$ and must be lower than $n$ (this gives the minimum number of landmarks). The solution of the minimization problem can now be written as:

$$
z(\mathbf{x})=\sum_{i=1}^{M} a_{i} \phi_{i}(\mathbf{x})+\sum_{i=1}^{n} w_{i} U_{i}(\mathbf{x})
$$

with some basis functions $U_{i}=U\left(\cdot, \mathbf{p}_{i}\right)$ depending on i) the dimension $d$ of the domain, ii) the order $m$ of the functional $J$ to be minimized and iii) the Hilbertspace $H$ of admissible functions $[5,15]$. If we choose the Sobolev space $H=\mathcal{H}^{2}$, we obtain the kernel

$$
U(\mathbf{x}, \mathbf{p})=\left\{\begin{array}{cc}
\theta_{m, d}|\mathbf{x}-\mathbf{p}|^{2 m-d} \ln |\mathbf{x}-\mathbf{p}| & 2 m-d \text { even positive integer } \\
\theta_{m, d}|\mathbf{x}-\mathbf{p}|^{2 m-d} & \text { otherwise }
\end{array}\right.
$$

with $\theta_{m, d}$ as defined in [17]. Note, that the basis functions $U_{i}$ span an $n$-dimensional space of functions that depends only on the source landmarks.

The coefficient vectors $\mathbf{a}=\left(a_{1}, \ldots, a_{M}\right)^{T}$ and $\mathbf{w}=\left(w_{1}, \ldots, w_{n}\right)^{T}$ can be computed through the following system of linear equations:

$$
\begin{array}{r}
\mathbf{K} \mathbf{w}+\mathbf{P a}=\mathbf{v} \\
\mathbf{P}^{T} \mathbf{w}=0,
\end{array}
$$

where $\mathbf{v}$ is the column vector of one component of the coordinates of the target points $\mathbf{q}_{i}$, and $K_{i j}=U_{i}\left(\mathbf{p}_{j}\right), P_{i j}=\phi_{j}\left(\mathbf{p}_{i}\right)$.

\section{Thin-Plate Spline Approximation Based on Regularization Theory}

To take into account landmark localization errors one has to weaken the interpolation condition (1). This can be done by combining an approximation criterion with the functional in (2). In the simplest case of a quadratic approximation term, this results in the following functional [17]:

$$
J_{\lambda}(\mathbf{u})=\sum_{i=1}^{n}\left|\mathbf{q}_{i}-\mathbf{u}\left(\mathbf{p}_{i}\right)\right|^{2}+\lambda J_{m}^{d}(\mathbf{u})
$$

Such functionals have been used for the reconstruction of surfaces from sparse depth data. Arad et al. [1] recently used a 2D approximation approach of this kind to represent and modify facial expressions. The first term (data term) measures the sum of the quadratic Euclidean distances between the transformed source landmarks and the target landmarks. The second term measures the smoothness of the resulting transformation. Hence, the minimization of (5) yields a transformation $\mathbf{u}$, which i) approximates the distance of the source landmarks 


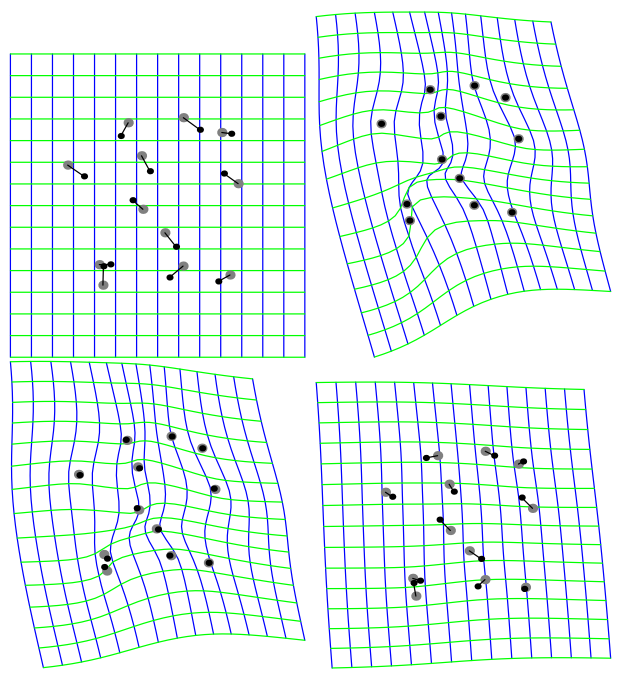

Fig. 1. Thin-plate spline approximation (input data, $\lambda=0,0.001$, and 0.1 ).

to the target landmarks and ii) is sufficiently smooth. The relative weight between the approximation behavior and the smoothness of the transformation is determined by the regularization parameter $\lambda>0$. If $\lambda$ is small, we obtain a solution with good approximation behavior (in the limit of $\lambda \rightarrow 0$ we have an interpolating transformation). If $\lambda$ is large, we obtain a very smooth transformation with little adaption to the local structure of the distortions. In the limit of $\lambda \rightarrow \infty$ we get a global polynomial of order up to $m-1$, which has no smoothness energy $J_{m}^{d}$ at all.

The interesting fact is that the solutions to the approximation problem have always the same form as in the case of interpolation. Also, the computational scheme is nearly the same. We only have to add $\lambda$ in the diagonal of the matrix $\mathbf{K}$ :

$$
\begin{aligned}
(\mathbf{K}+\lambda \mathbf{I}) \mathbf{w}+\mathbf{P a} & =\mathbf{v} \\
\mathbf{P}^{T} \mathbf{w} & =0
\end{aligned}
$$

Adding $\lambda$ in the diagonal also results in a better conditioned system of linear equations than in the case of interpolation.

Fig. 1 shows an example of the thin-plate spline approximation scheme in two dimensions (with $m=2$ ) for different values of $\lambda$. The small black points and big grey points mark the positions of the source and target landmarks, respectively. The top-left part of Fig. 1 shows the regular grid. The top-right part shows the result for $\lambda=0$, which is equivalent to the interpolation scheme. At some locations the grid is heavily distorted, especially around the two close landmarks in the bottom-left part of this grid. The two bottom grids visualize results for $\lambda=0.001$ (bottom-left) and $\lambda=0.1$ (bottom-right), where the latter one nearly yields a pure affine transformation. 
A generalization of the approximation scheme can be made, if we have information about the expected accuracy of the landmarks. Then, we can weight each single data term $\left|\mathbf{q}_{i}-\mathbf{u}\left(\mathbf{p}_{i}\right)\right|^{2}$ by the inverse variance $1 / \sigma_{i}^{2}$. If the variance is high, i.e. landmark localization is uncertain, then less penalty is given to the approximation error at this point. The data term now reads

$$
\sum_{i=1}^{n} \frac{\left|\mathbf{q}_{i}-\mathbf{u}\left(\mathbf{p}_{i}\right)\right|^{2}}{\sigma_{i}^{2}}
$$

and we have to solve the following system of equations:

$$
\begin{aligned}
\left(\mathbf{K}+\lambda \mathbf{W}^{-1}\right) \mathbf{w}+\mathbf{P a} & =\mathbf{v} \\
\mathbf{P}^{T} \mathbf{w} & =0,
\end{aligned}
$$

where $\mathbf{W}=\operatorname{diag}\left\{1 / \sigma_{1}^{2}, \ldots, 1 / \sigma_{n}^{2}\right\}$. Note, that this approach can be applied to images of arbitrary dimension, i.e. in particular to $2 \mathrm{D}$ as well as $3 \mathrm{D}$ images.

\section{Experimental Results}

Within the scenario of CT-MR registration as discussed above we here consider the important application of correcting patient-induced susceptibility distortions of MR images. To this end we have acquired two sagittal MR images of a healthy human volunteer brain with typical susceptibility distortions. In our experiment we used a high-gradient MR image as "ground truth" (instead of clinically common CT images) to avoid exposure of the volunteer to radiation. Both turbo-spin echo images have consecutively been acquired on a modified Philips $1.5 T$ MR scanner with a slice thickness of $4 \mathrm{~mm}$ without repositioning. Therefore, we are sure that we actually have identical slicing in space. Using a gradient of $1 \mathrm{mT} / \mathrm{m}$ and $6 \mathrm{mT} / \mathrm{m}$ for the first and second image then leads to a shift of ca. $7.5 \ldots 10 \mathrm{~mm}$ and ca. $1.3 \ldots 1.7 \mathrm{~mm}$, respectively (see [14] for details). In our example, we use the second image as "ground truth" to demontrate that the elastic matching approach can cope with these distortions and that the use of approximating thin-plate splines is advantageous.

Within each of the two images we have interactively selected 20 point landmarks. To simulate outliers, one of the landmarks in the first image (No. 3) has been shifted about 15 pixels away from its true position for demonstration purposes (see Fig. 2). Note, however, that interactive localization of landmarks actually can be prone to relatively large errors. Fig. 3 shows the results of the interpolating vs. the approximating $(\lambda=0.015)$ thin-plate spline approach. Each result represents the transformed first image. In the difference image of the two results in Fig. 4 we see that the largest differences occur at the shifted landmark No. 3 which is what we expect. In Fig. 3 on the left it can be seen that the interpolation scheme yields a rather unrealistic deformation since it forces all landmark pairs, including the pair with the simulated outlier, to exactly match each other. Using our approximation scheme instead yields a more accurate registration result (Fig. 3 on the right). The increased accuracy can also be demonstrated by 

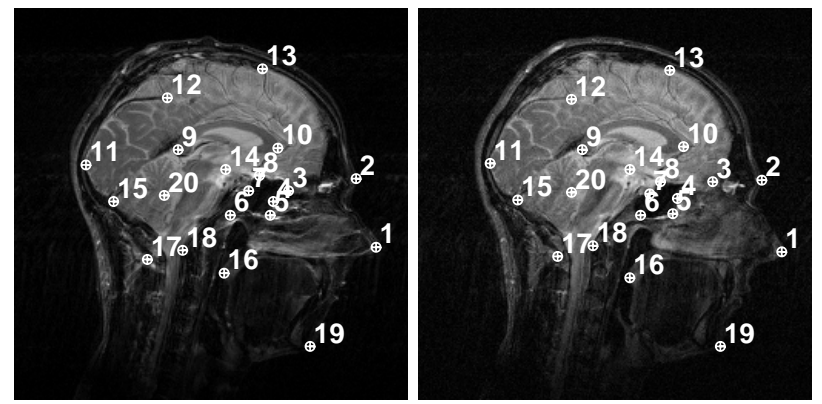

Fig. 2. Original MR images with landmarks: First (left) and second (right) image.
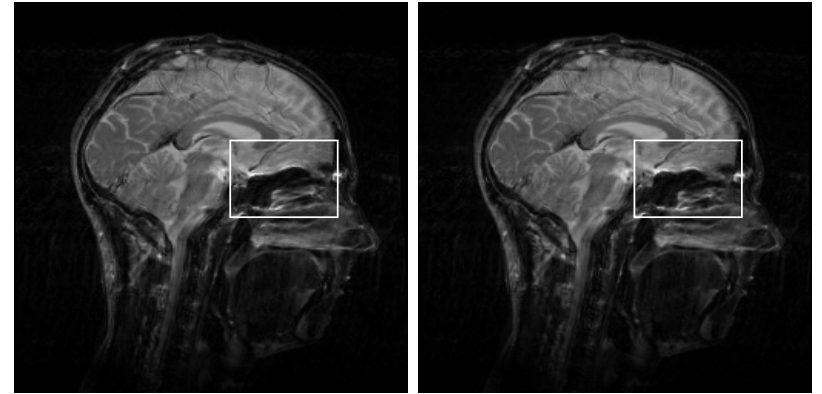

Fig. 3. Registration results: Interpolation (left) and approximation (right).

computing the distance between the grey-value edges of the transformed images and those of the second image. In our case, we applied a distance transformation [12] to Canny edges. The results for the marked rectangular image parts in Fig. 3 can be seen in Fig. 5. Here, the intensities represent the registration error, i.e., the brighter the larger is the error. In particular at the marked circular areas, which indicate the grey-value edges perpendicular to the simulated shift, we see that the registration accuracy has increased significantly. Note, that in this experiment we only used equal weights for all landmarks. However, incorporation of quantitative knowledge about the landmark uncertainties promises a further increase of accuracy.

\section{Semi-Automatic Landmark Localization}

The problem with point landmarks is their reliable and accurate extraction from $3 \mathrm{D}$ images. Therefore, 3D point landmarks have usually been selected manually (e.g., Evans et al. [6], Hill et al. [8]). Only a few automatic approaches have been proposed (e.g., Thirion [16]). In this section, we briefly describe our first investigations into semi-automatic localization of 3D anatomical point landmarks. Semi-automatic means that either a region-of-interest (ROI) or an approximate 


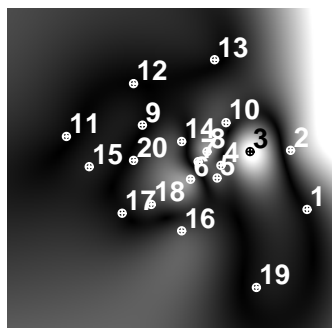

Fig. 4. Difference between the two registration results.

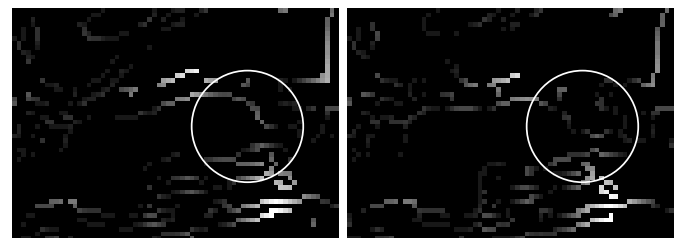

Fig. 5. Registration errors: Interpolation (left) and approximation (right).

position of a specific landmark (or both) is given by the user. Then, an algorithm has to provide a refined position of the landmark. Alternatively, landmark candidates for a large ROI or even for the whole data set may be provided automatically from which the final set of landmarks is selected interactively. Such a semi-automatic approach has the advantage that a user has the possibility to control the results ("keep-the-user-in-the-loop").

Within a ROI we apply specific $3 \mathrm{D}$ differential operators such as to exploit the knowledge about a landmark as far as possible, in particular it's geometric structure. Typical landmark types in $3 \mathrm{D}$ are blobs, line-plane intersections and curvature extrema. Blobs and line-plane intersections can be determined by exploiting the Eigenvalues of the Hessian matrix $\underline{H}_{g}$. To localize curvature extrema we exploit the two principal curvatures of the isocontour as is also done in approaches for detecting 3D ridge lines. In our first experiments we used an operator which represents the Gaussian curvature, i.e. the product of the two principal curvatures $K=\lambda_{1} \lambda_{2}$, multiplied with the fourth power of the gradient magnitude $|\nabla g|$. Fig. 6 shows a result of this operator for the right frontal horn in a $3 \mathrm{D}$ MR data set. It can be seen that we obtain a strong operator response at the tip of the frontal horn.

We have also investigated 3D differential operators for providing a set of landmark candidates. Since we want to apply these operators on a large ROI or even on the whole data set, it is indispensable to use computationally efficient schemes. To this end we have extended existing 2D differential operators for detecting points of high intensity variations ('corner detectors') to 3D. For a recent analytic study of such 2D operators see Rohr [13]. These operators have the advantage that only low order partial derivatives of the image function are necessary (first or first and second order). Therefore, these operators are computationally efficient and do not suffer from instabilities of computing high order partial derivatives. As an example, in Fig. 7 we show the application of the 2D operator of Förstner [7] vs. a 3D extension of it: $\operatorname{det} \underline{C}_{g} /$ trace $\underline{C}_{g} \rightarrow \max$, where $\underline{C}_{g}=\overline{\nabla g(\nabla g)^{T}}$ and $\nabla g$ denotes the image gradient in $2 \mathrm{D}$ and $3 \mathrm{D}$, respectively. Note, that in the $2 \mathrm{D}$ case many well detected landmarks agree with the interactively selected landmarks in Bookstein [3]. Note also, that the 3D operator actually takes into account the 3D structure of the landmarks and therefore in a 

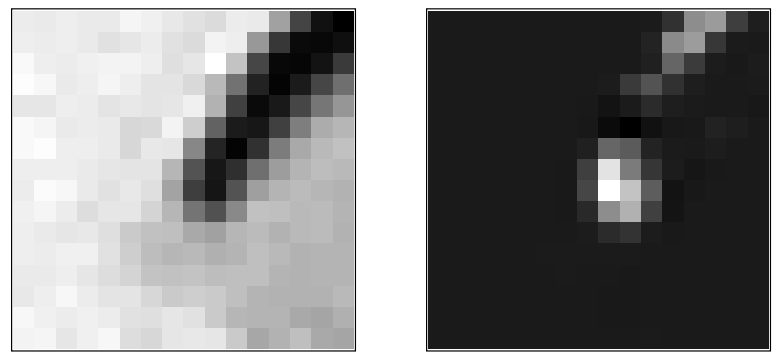

Fig. 6. Right frontal horn in a 3D MR data set (left) and result of computing the 3D Gaussian curvature (right)
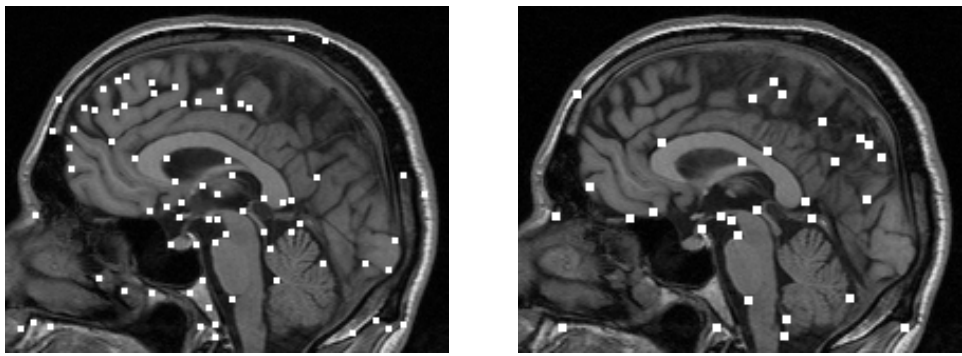

Fig. 7. Landmark candidates: Application of a 2D 'corner' detector (left) vs. a 3D extension (right) on a 2D and $3 \mathrm{D}$ MR image, respectively.

single slice of a $3 \mathrm{D}$ image only a few of the $3 \mathrm{D}$ point landmarks are visible, i.e., other landmarks according to [3] have been detected in different slices.

\section{Summary}

We have introduced an approximating thin-plate spline approach based on regularization theory to elastic registration of medical image data. In the case of landmark localization errors this scheme generally yields a more accurate and robust registration result. In particular large localization errors, i.e. outliers, do not affect the registration result as much as is the case with an interpolation scheme. In addition, we have reported on investigations into semi-automatic extraction of anatomical point landmarks from $2 \mathrm{D}$ as well as $3 \mathrm{D}$ tomographic images of the human brain.

\section{Acknowledgement}

Support of Philips Research Hamburg, project IMAGINE (IMage- and Atlas-Guided Interventions in NEurosurgery), and of the E.U., project EASI, is gratefully acknowledged. We would like to thank K. Jungnickel (formerly with Philips Research Hamburg) for providing us with the MR images showing the susceptibility distortions. 


\section{References}

1. N. Arad, N. Dyn, D. Reisfeld, and Y. Yeshurun. Image warping by radial basis functions: Application to facial expressions. Computer Vision, Graphics, and Image Processing, 56(2):161-172, 1994.

2. F. Bookstein. Principal warps: Thin-plate splines and the decomposition of deformations. IEEE Trans. Pattern Anal. and Machine Intell., 11(6):567-585, 1989.

3. F. Bookstein. Thin-plate splines and the atlas problem for biomedical images. In A. Colchester and D. Hawkes, editors, 12 th Internat. Conf. Information Processing in Medical Imaging, volume 511 of Lecture Notes in Computer Science, pages 326342, Wye/UK, 1991. Springer-Verlag Berlin Heidelberg.

4. H. Chang and J. Fitzpatrick. A technique for accurate magnetic resonance imaging in the presence of field inhomogeneities. IEEE Trans. Med. Imaging, 11:319, 1992.

5. J. Duchon. Interpolation des fonctions de deux variables suivant le principle de la flexion des plaques minces. R.A.I.R.O. Analyse Numérique, 10(12), 1976.

6. A. Evans, W. Dai, L. Collins, P. Neelin, and S. Marrett. Warping of a computerized 3-d atlas to match brain image volumes for quantitative neuroanatomical and functional analysis. In M. Loew, editor, Medical Imaging V: Image Processing, volume 1445 of Proc. SPIE, pages 236-246, San Jose, CA, 1991.

7. W. Förstner. A feature based correspondence algorithm for image matching. Intern. Arch. of Photogrammetry and Remote Sensing, 26-3/3:150-166, 1986.

8. D. Hill, D. Hawkes, J. Crossman, M. Gleeson, T. Cox, E. Bracey, A. Strong, and P. Graves. Registration of MR and CT images for skull base surgery using pointlike anatomical features. The British J. of Radiology, 64(767):1030-1035, 1991.

9. K. Lüdeke, P. Röschmann, and R. Tischler. Susceptibility artifacts in nmr imaging. MRI, 3:329, 1985.

10. K. Mardia and J. Little. Image warping using derivative information. In F. Bookstein, J. Duncan, N. Lange, and D. Wilson, editors, Mathematical Methods in Medical Imaging III, volume 2299 of Proc. SPIE, pages 16-31, San Diego, CA, 25-26 July 1994.

11. J. Michiels, H. Bosmans, P. Pelgrims, D. Vandermeulen, J. Gybels, G. Marchal, and P. Suetens. On the problem of geometric distortion in magnetic resonance images for stereotactic neurosurgery. Mag. Res. Imag., 12:749, 1994.

12. D. W. Paglieroni. A unified transform algorithm and architecture. Machine Vision and Applications, 5(1):47-55, 1992.

13. K. Rohr. Localization properties of direct corner detectors. J. of Mathematical Imaging and Vision, 4(2):139-150, 1994.

14. K. Rohr, H. S. Stiehl, R. Sprengel, W. Beil, T. M. Buzug, J. Weese, and M. H. Kuhn. Point-based elastic registration of medical image data using approximating thin-plate splines. Techn. Report FBI-HH-M-254/96, FB Informatik, Universität Hamburg, Febr. 1996.

15. R. Sprengel, K. Rohr, and H. S. Stiehl. Thin-plate spline approximation for image registration. 1996. submitted for publication.

16. J.-P. Thirion. Extremal points: definition and application to $3 d$ image registration. In Proc. IEEE Conf. on Computer Vision and Pattern Recognition, pages 587-592, Seattle/Washington, USA, 1994.

17. G. Wahba. Spline Models for Observational Data. Society for Industrial and Applied Mathematics, Philadelphia, Pennsylvania, 1990.

This article was processed using the IATEX macro package with LLNCS style 